Scripta METALLURGICA

et MATERIALIA
Vo1. 24, pp. 101-106, 1990

Printed in the U.S.A.
Pergamon Press plc

All rights reserved

\title{
EFFECTS OF PARTICLE SIZE ON INHIBITED GRAIN GROWTH
}

\author{
G. N. Hassold*†, E. A. Holm*, and D. J. Srolovitz* \\ *Dept. of Materials Science and Engineering, University of Michigan, Ann Arbor, MI 48109; †Dept. of Science and \\ Mathematics, GMI Engineering and Management Institute, Flint, MI 48504
}

(Received October 24, 1989)

\section{Introduction}

Inert, second-phase particles are often dispersed in metallic and ceramic materials to control grain morphology and size [1,2]. The microstructure imposed by the particles affects the final properties of the material. For instance, dispersions of particles have long been known to pin grain boundaries and inhibit grain growth [3].

Many theoretical studies have examined the grain boundary pinning effect of randomly dispersed, monosized, isotropic particles. Most of these studies are based on the Zener approach to inhibited grain growth, in which the pinning force exerted by spherical particles on a randomly positioned grain boundary is equated with the grain boundary curvature driving force for grain growth [4]. For particle inhibited grain growth in three dimensions, the Zener equation may be generalized as

$$
\frac{D_{B}}{r} \propto \frac{1}{f}
$$

where $D_{g}$ is the average pinned grain diameter, $r$ is the radius of the pinning particles, and $f$ is the volume fraction of the particles $\{5,6]$.

The Zener assumptions fail, however, if the intersections of grain boundaries with pinning particles are nonrandom. For instance, if the second phase particles pin the grain boundaries strongly, the grain boundaries may deform into the shapes that maximize the number of particle-boundary intersections [6-8]. In this case, pinning occurs when

$$
\mathrm{V}_{\mathrm{g}} \cdot \mathrm{N}_{\mathrm{p}} \propto \mathrm{n}_{\mathrm{c}}
$$

where $V_{g}$ is the average grain volume, $N_{p}$ is the number of pinning particles per unit volume, and $n_{c}$ is the number of particles which stabilizes the average grain. Thus, since $V_{g^{\alpha}} D_{g}{ }^{d}$ and $N_{p e c} f / r^{d}$,

$$
\frac{D_{\mathrm{g}} \times \frac{1}{\mathrm{r}}}{\mathrm{f}^{1 / \mathrm{d}}}
$$

where $d$ is the dimensionality of the system. Expressions for $n_{c}$ have been derived by Srolovitz, et al. [7], Doherty, et al. [8] and Hillent [6]. Altematively, this expression may be derived by assuming that the grain diameter is proportional to the distance between pinning particles in d dimensions, $\Delta_{d}$. Since $\Delta_{d}$ os $f^{-1 / d}[9]$, if $D_{g} \propto \Delta_{d}$, Eq. (3) follows directly.

Srolovitz, et al. [7] performed Monte Carlo computer simulations of two-dimensional, pinned grain growth by mapping the two-dimensional microstructure onto a discrete lattice. Anderson, et al. [10] later performed similar simulations in three dimensions. Both simulations reproduced the dependence of $D_{g}$ on $f$ predicted in $E q$. (3). Since the particles in both studies were of size equal to one lattice site $(r=1)$, it has been speculated that the disagreement with Eq. (1) is simply a consequence of the discrete lattice used in the simulation. Further, since single-site particles have a size on the order of the "width" of the grain boundary in the discrete lattice, questions have been raised about the validity of generalizing the $r=1$ results to $r>1$.

The purpose of this communication is to re-examine pinned grain growth simulation results [7] for a range of particle sizes.

\section{Computer Simulation}

The Monte Carlo model for the simulation of normal grain growth has previously been described in theoretical and computational detail [7,11-13]. A continuum microstructure is mapped onto a triangular lattice by assigning each lattice site an index corresponding to the orientation of the grain in which it is embedded. Sites with one or more unlike 
nearest neighbors are boundary sites; sites with six like nearest neighbors are bulk (or interior) sites. The grain boundary energy at a site is specified by assigning a positive energy to boundary sites and zero energy to interior sites, and is computed via the Hamiltonian

$$
H=-J \sum_{m n}\left(\delta_{s_{i} s_{j}}-1\right)
$$

where $\delta \mathrm{S}_{\mathrm{i}} \mathrm{S}_{\mathrm{j}}$ is the Kronecker delta, the sum is taken over nearest neighbor sites (nn), and $\mathrm{J}$ is a positive constant which scales the energy.

Grain growth kinetics are determined through a Monte Carlo technique. First, a lattice site and an orientation are chosen at random. The orientation of the chosen site is then changed to the random orientation if and only if the energy change for the reorientation is negative or zero. Since only negative or zero energy are allowed, the implicit Monte Carlo temperature of the simulation is $T=0$. Previous studies have determined that growth simulations at $T=0$ and at finite $T$ yield similar results $[7,11]$. In the Monte Carlo simulation, time is incremented after each attempted reorientation by $\left(1 / \mathrm{N}_{\mathrm{act}}\right)$ Monte Carlo steps (MCS) where $\mathrm{N}_{\mathrm{act}}$ is the number of active (non-particle) lattice sites.

Inert particles are incorporated into the simulation by choosing sites at random in the lattice and assigning these sites an index not equal to any of the grain orientation indices. For particles of $>1$, the first through $n^{\text {th }}$ nearest neighbor sites are also assigned the "non-active" orientation index. If two randomly placed particles were found to overlap, the particle area fraction was modified to account for the double counting. The particle-matrix interface energy is computed in the same manner as the grain boundary energy; hence, the interface energy is nearly isotropic. Since particle sites are not re-oriented during the simulation, particles are immobile and of a fixed size.

In these studies, the initial grain orientations were determined by assigning each grain site a random orientation. Then, the microstructures were allowed to evolve until no further growth was detected (generally at about $10^{6} \mathrm{MCS}$ ). The numerical data given are averages of ten independent runs on a 40,000 site lattice. The particles employed in the present study were all hexagonal and ranged in size from one to 61 sites.

\section{Results and Discussion}

Equation (3) predicts that at constant area fraction of particles, $D_{g} / r$ is constant; thus, changing the particle size should lead to a proportional change in the pinned grain size. This is reasonable, since increasing the particle size essentially rescales all of the lengths in the simulation. Therefore, the data for all particle sizes should fall on the curve specified by Eq. (3).

Figure 1 shows a plot of $D_{g} / r$ versus $1 / f$ for various particle sizes and area fractions. The linearity of the data on the $\log$-log scale indicates a power law relationship

$$
\frac{\mathrm{D}_{\mathrm{g}}}{\mathrm{r}} \times \mathrm{k}\left(\frac{1}{\mathrm{f}}\right)^{\mathrm{m}}
$$

for $\mathrm{f}<0.1$. A best fit to the data for $\mathrm{f}<0.1$ gives $\mathrm{k}=1.197 \pm 0.070$ and $\mathrm{m}=0.547 \pm 0.009$ with a correlation coefficient of 0.9967 . This relationship is in good agreement with Eq. (3) for $\mathrm{d}=2$; thus, the two-dimensional simulation results for single lattice site particles [7] are valid for a wide range of particle sizes. Moreover, since mapping the pinning particles onto a discrete lattice had no effect on the applicability of $\mathrm{Eq}$. (3) for $\mathrm{d}=2$, the three dimensional simulation results for single site particles [10] are likewise expected to be valid for all particle sizes.

In the range $0.002 \leq f \leq 0.02$, a very slight systematic dependence of $D_{g} / r$ on $r$ may be observed: As $r$ increases, $\mathrm{D}_{\mathrm{g}} / \mathrm{r}$ decreases very slightly at a constant $\mathrm{f}$. This behavior does not show a limitation in Eq. (3); instead, it is a result of the finite sample size used in the simulation. Since a grain may not be larger than the lattice itself, the grain size distribution [11,12] is truncated at a grain size of $D_{g}=\mathrm{N}^{1 / 2}$, where $\mathrm{N}$ is the total number of sites in the lattice. However, the exclusion of a few very large grains decreases the mean grain size, and relatively more grains are excluded from the distribution as the mean grain size approaches $\mathrm{N}^{1 / 2}$. Because the mean grain size increases with particle size, as $\mathrm{r}$ increases at a constant $f, D_{g} / \mathrm{r}$ decreases slightly.

The deviation from linearity for $\mathrm{f}>0.1$ may be attributed to the formation of clusters of overlapping particles as the volume fraction of particles increases. These clusters are not isotropic and their effective size is greater than $r$. Therefore, they may be expected to inhibit grain growth with a functionality different from Eq. (3).

As in the previous study [7], the inhibited grain growth kinetics were found to have two distinct regimes. Initially, growth follows normal grain growth kinetics, with $\mathrm{D}_{\mathrm{g} \alpha \mathrm{t}^{\mathrm{n}}}$ (power law growth). In agreement with previous results for early time grain growth [7,11], $n$ was found to be approximately 0.4 for all $r$ and $f$. (In the long time limit, the grain growth simulations yield $n=0.5[11,12]$.) The second growth regime is characterized by a pinned 
microstructure with $D_{g}$ constant. Because the transition between the two regimes is quite abrupt, a cutoff time $\left(t_{c}\right)$ may be defined as the time at which power-law growth ceases and the final grain radius is reached. Since the shapes of all growth curves are identical, a scaling which causes the cutoff times to correspond should collapse all of the growth kinetics to a single curve.

Since $D_{g^{\alpha} t^{n}}$ and $D_{g} \propto r / f^{m}$ at $t_{c}, D_{g}$ should scale with $\left(\mathrm{fm}^{\mathrm{m}} / \mathrm{r}\right)$ and $t$ should scale with $\left(\mathrm{f}^{\mathrm{m} / \mathrm{r})^{1 / n}}\right.$. In this study, $n=0.4$ and $m=0.55$. Figure 2 shows a scaled plot of $D_{g}$ versus $t$ for four different particle sizes and area fractions. Clearly, this scaling provides an excellent fit to all of the simulation data.

Figure 3 shows pinned microstructures for $\mathrm{f}=0.02$ and various particle radii. It is apparent that most particles are located on the grain boundaries, in agreement with the $r=1$ case [7]. Because the particles strongly pin the grain boundaries, the grain boundaries are highly non-random in location; for instance, note the irregularity of the boundary of the large grain in the lower right comer of Fig. 3(a). Also interesting to note is that only two particles are needed to stabilize the small grain between the two large grains in the center of Fig. 3(c). As expected from Eq. (3), the pinned grain size decreases with decreasing particle size at constant $f$.

Figure 4 shows simulated pinned microstructures for various particle area fractions and $r=7$. It is again clear that in all cases, most of the particles are located on grain boundaries. Because particles are more closely spaced as the volume fraction increases, the pinned grain size decreases as the volume fraction of particles increases.

\section{Conclusions}

1. A Monte Carlo Potts model computer simulation of two-dimensional grain growth inhibited by randomly dispersed, monosized particles has reproduced the "strong pinning" growth relationship

$$
\frac{D_{g}}{r} \propto \frac{1}{f^{1 / d}}
$$

for $r \geq 1$ and $f<0.10$. Above $f=0.10$, this relationship fails due to the formation of particle clusters.

2. The result that single site particles follow the same scaling laws as large particles suggest that the results of earlier two- and three-dimensional simulations, which employed single site particles, are valid.

3. Inhibited grain growth is characterized by two kinetic regimes: a normal growth regime with $\mathrm{D}_{\mathrm{g}}{ }^{\mathrm{n}} \mathrm{f}^{\mathrm{n}}$ followed by a pinned regime with $D_{g}$ constant. Thus, $D_{g}$ scales with $(f m / r)$, and $t$ scales with $\left(f^{m} / r\right)^{1 / n}$ where $m=0.55$ and $n=0.40$ in the present simulations.

4. The two-dimensional microstructures generated by the simulation display the characteristics of a strongly pinned structure, with nearly all of the pinning particles located on the grain boundaries.

\section{Acknowledgements}

EAH's work has been supported in part by a National Science Foundation Graduate Research Fellowship. The authors gratefully acknowledge helpful conversations with Dr. A. D. Rollett.

\section{Beferences}

1. R. E. Reed-Hill, Physical Metallurgy Principles, 2nd edition, p. 311, Brooks/Cole Engineering Division, Monterey, CA (1973).

2. W. D. Kingery, H. K. Bowen, and D. R. Uhlmann, Introduction to Ceramics, 2nd edition, pp. 469-490, John Wiley and Sons, New York (1976).

3. Z. Jeffries and R. S. Archer, The Science of Metals, p. 95, McGraw-Hill Book Co., New York (1924).

4. C. S. Smith, Trans, AIME, 17515 (1949).

5. M. Hillert, "Zener's Pinning Effect - Role of Dispersed Particles on Grain Size Control," preprint.

6. M. Hillert, Acta Metall, 363177 (1988).

7. D. J. Srolovitz, M. P. Anderson, G. S. Grest, and P. S. Sahni, Acta Metall. 32[9] 1429 (1984).

8. R. D. Doherty, D. J. Srolovitz, A. D. Rollett, and M. P. Anderson, Scripta Metall, 21675 (1987).

9. E. E. Underwood, Quantitative Stereology, p. 85, Addison-Wesley Publishing Co., Reading, MA (1970). 
10. M. P. Anderson, G. S. Grest, R. D. Doherty, K. Li, and D. J. Srolovitz, Scripta Metall, 23753 (1989).

11. M. P. Anderson, D. J. Srolovitz, G. S. Grest, and P. S. Sahni, Acta.Metall, 32[5] 783 (1984).

12. D. J. Srolovitz, M. P. Anderson, P. S. Sahni, and G. S. Grest, Acta Metall. 32[5] 793 (1984).

13. G. S. Grest, M. P. Anderson, and D. J. Srolovitz, Phys. Rey. B. 38[7] 4752 (1988).

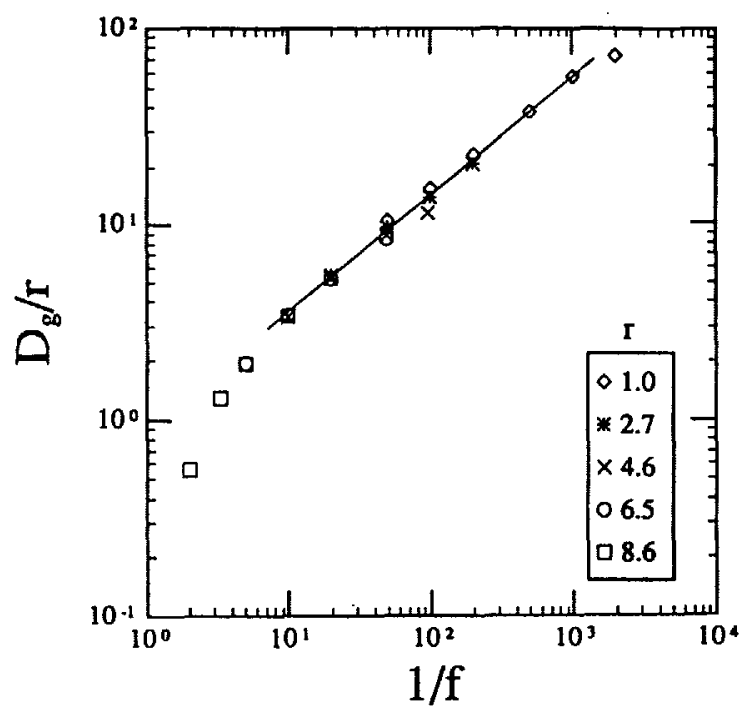

Eigure 1 The effect of particle volume fraction on pinned grain size in two dimensions. $\mathrm{D}_{\mathrm{g}}$ is the pinned grain diameter, $r$ is the pinning particle radius, and $f$ is the particle volume fraction. For $f<0.1$, the curve behaves as predicted for strong pinning by isotropic particles. For $1>0.1$, the formation of particle clusters causes a deviation from the predicied behavior.

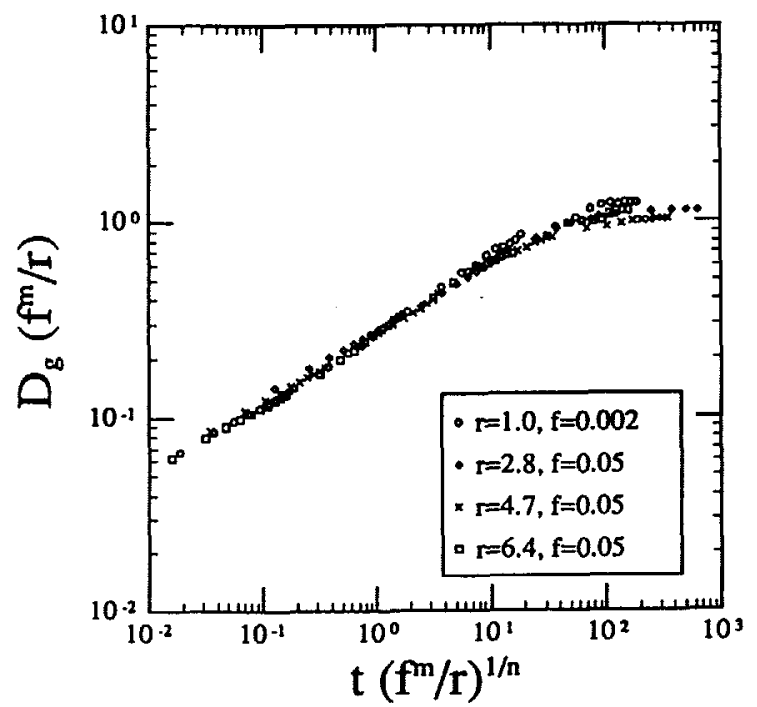

Figure 2. The kinetics of inhibited grain growth for various pinning particle sizes and volume fractions. $\mathrm{D}_{\mathrm{g}}$ is the pinned grain diameter, $r$ is the pinning particle radius, $f$ is the particle volume fraction, and $t$ is the time in Monte Carlo steps. Growth first follows a normal grain growth curve, then ceases at some cutoff time when the grains become fully pinned. The data scale when the cutoff times are forced to coincide. 


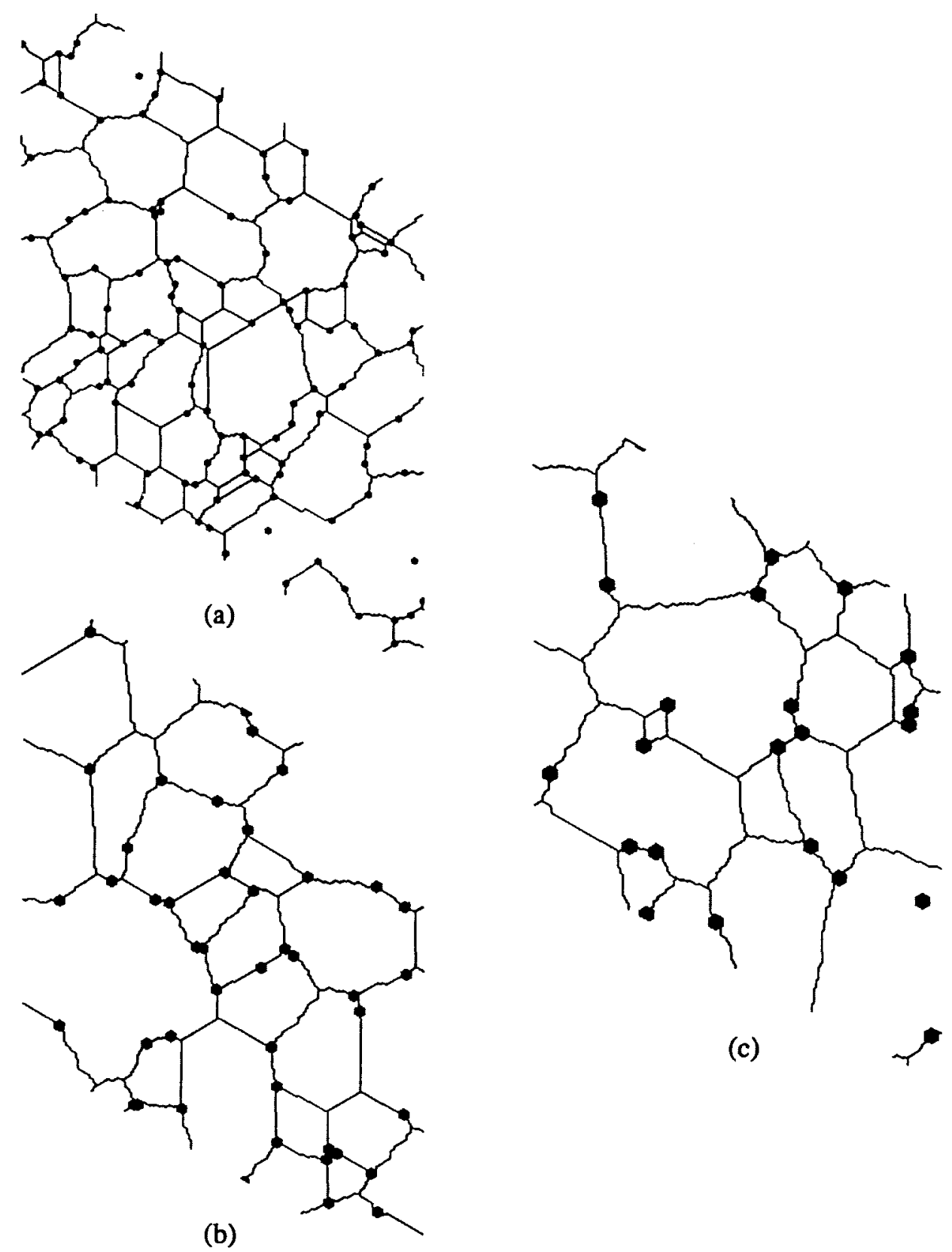

Figure 3. Computer generated pinned microstructures for $f=0.02$ with various particle sizes. (a) $r=2.67$ (b) $r=4.59$ (c) $r=6.22$. The grain boundaries are strongly pinned by the particles, so as $r$ increases the grain diameter increases. 

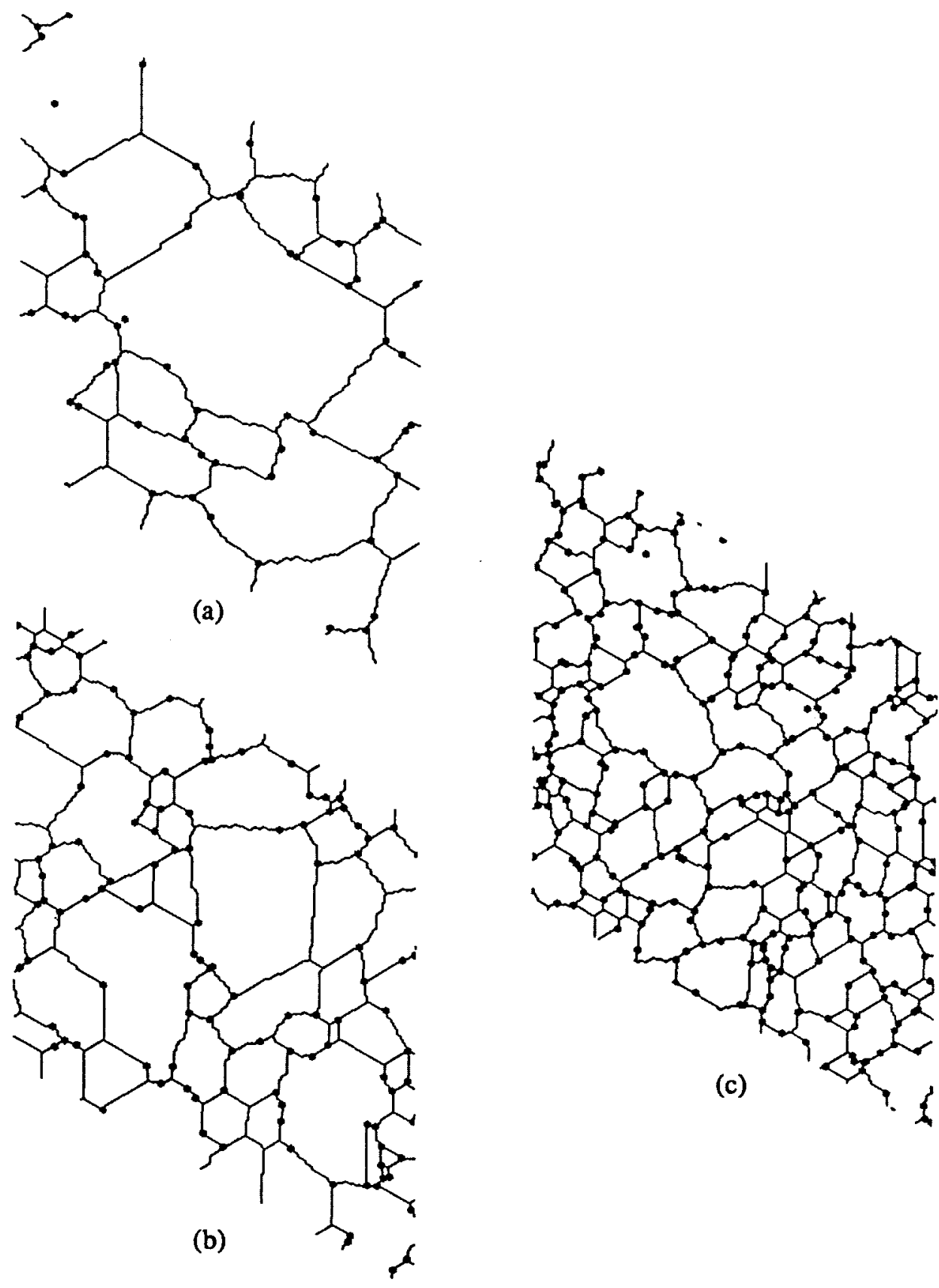

Figure 4. Computer generated pinned microstructures for $\mathrm{s}=2.67$ with various particle fractions. (a) $\mathrm{f}=0.01$ (b) $f=0.02$ (c) $f=0.05$. The grain boundaries are strongly pinned by the particles, so as $f$ increases the grain diameter decreases. 